\title{
Quantum Backaction Cancellation in the Audio Band
}

\author{
Jonathan Cripe $\odot,{ }^{1, *}$ Torrey Cullen, ${ }^{1}$ Yanbei Chen, ${ }^{2}$ Paula Heu, ${ }^{3}$ David Follman $\odot,{ }^{3, *}$ \\ Garrett D. Cole $\oplus^{3,4, \$}$ and Thomas Corbitt $\circledast^{1, \dagger}$ \\ ${ }^{1}$ Department of Physics and Astronomy, Louisiana State University, Baton Rouge, Louisiana 70803, USA \\ ${ }^{2}$ Theoretical Astrophysics 350-17, California Institute of Technology, Pasadena, California 91125, USA \\ ${ }^{3}$ Crystalline Mirror Solutions LLC and GmbH, \\ Santa Barbara, California 93101, USA and 1010 Vienna, Austria \\ ${ }^{4}$ Vienna Center for Quantum Science and Technology (VCQ), Faculty of Physics, University of Vienna, \\ A-1090 Vienna, Austria
}

(Received 27 August 2019; revised 11 June 2020; accepted 31 July 2020; published 23 September 2020)

\begin{abstract}
We report on the cancellation of quantum backaction noise in an optomechanical cavity. We perform measurements of the displacement of the microresonator, one in reflection of the cavity and one in transmission of the cavity. We show that measuring the amplitude quadrature of the light transmitted by the optomechanical cavity allows us to cancel the backaction noise between 2 and $50 \mathrm{kHz}$ as a consequence of the strong optical spring present in the detuned cavity. This cancellation yields a more sensitive measurement of the microresonator's position with a $2 \mathrm{~dB}$ increase in sensitivity. To confirm that the backaction is eliminated, we measure the noise in the transmission signal as a function of circulating power and use a correlation technique between two photodetectors to remove shot noise. Remaining backaction noise would be observable as a power-dependent noise floor, which is not observed. Eliminating the effects of backaction in this frequency regime is an important demonstration of a technique that could be used to mitigate the effects of backaction in interferometric gravitational wave detectors such as Advanced LIGO, VIRGO, and KAGRA.
\end{abstract}

DOI: 10.1103/PhysRevX.10.031065

Subject Areas: Optics, Quantum Physics

\section{INTRODUCTION}

Over the past century, interferometers have been used to perform increasingly sensitive measurements for a wide variety of applications. From the pioneering work of Michelson and Morley's attempts to measure ether [1] to the recent discoveries of gravitational waves by the worldwide gravitational wave detector network [2-4], interferometers have been used to probe the minuscule displacements of mechanical objects. Shot noise, a consequence of the particle nature of light, has been a fundamental limitation to the sensitivity of gravitational wave interferometers. As the power employed in advanced gravitational wave detectors is increased in order to reduce the impact of shot noise, the detectors are approaching the regime in which quantum backaction (QBA) begins to limit

\footnotetext{
*jonathancripe90@gmail.com

tcorbitt@phys.lsu.edu

${ }^{\ddagger}$ Present address: Thorlabs Crystalline Solutions, Santa Barbara, CA, USA.

Published by the American Physical Society under the terms of the Creative Commons Attribution 4.0 International license. Further distribution of this work must maintain attribution to the author(s) and the published article's title, journal citation, and DOI.
}

the sensitivity. QBA arises from the fluctuations of the ponderomotive force imparted by the light used in the measurement [5-7]. Only recently, however, have experiments been able to observe the subtle quantum effects of QBA [8-11].

In a previous experiment utilizing the same optomechanical system as investigated here, we demonstrated that QBA was the dominant source of its motion between 10 and $50 \mathrm{kHz}$ [12]. A key element of this system is the low-loss, single-crystal microresonator [12-17] that forms one mirror of an optomechanical Fabry-Perot cavity. Here we build upon the previous results and demonstrate a method of canceling the QBA in a tabletop interferometer that serves as a test bed for quantum noise reduction schemes for future gravitational wave detectors.

With experiments now operating in the QBA-limited regime, ideas for manipulating and ultimately removing the effects of QBA have become experimentally accessible. Several recent experiments have successfully evaded QBA using two mechanical oscillators [18] and a joint measurement on a mechanical oscillator and atomic spin oscillator [19]. One proposed method of removing QBA in large-scale gravitational wave detectors is a variational measurement, in which the readout quadrature is chosen such that the measurement is free of QBA $[20,21]$. The variational readout technique utilizes correlations between 
quadratures of the light to effectively cancel the QBA, resulting in a measurement limited only by shot noise [22], which would result in an increase in sensitivity below $100 \mathrm{~Hz}$ when applied to a detector such as Advanced LIGO.

Ordinarily, this cancellation must be done in a frequency-dependent way using an optical filter cavity. In this work, we take advantage of the correlations created in a detuned optomechanical cavity with a strong optical spring to cancel the effects of QBA in a single, frequencyindependent quadrature, specifically the amplitude quadrature of the light that is transmitted through the cavity. We present two displacement measurements of the microresonator, one in reflection of the cavity and one in transmission, and show that the measurement performed in transmission is free of QBA. The transmission measurement shows a reduction of up to $2 \mathrm{~dB}$ in displacement noise between the frequencies of 2 and $50 \mathrm{kHz}$ compared to the reflection measurement. This reduction in displacement noise corresponds to an increase in the signal-to-noise ratio when compared to a displacement measurement where QBA is present.

\section{THEORY}

To understand how we are able to perform a QBA-free measurement of the position of the microresonator, consider the equation of motion of the movable mirror, given by

$$
\begin{aligned}
m \frac{d^{2} x}{d t^{2}} & =-\frac{2 \delta P}{c}+F_{\mathrm{ext}} \\
& =-\frac{2}{c}\left[\left(\frac{\partial P}{\partial a_{1}}\right)_{x} a_{1}+\left(\frac{\partial P}{\partial x}\right)_{a_{1}} x\right]+F_{\mathrm{ext}},
\end{aligned}
$$

where $m$ and $x$ are the mass and position of the microresonator, $\delta P$ is the power fluctuation, $F_{\text {ext }}$ is an external force, and $c$ is the speed of light. The first term in the brackets is the fluctuating radiation pressure acting on the mirror, driven by incoming vacuum fluctuations in the amplitude quadrature $a_{1}$. The second term arises in a detuned cavity and creates the optical spring effect [23]. Throughout this section, we are able to neglect the mechanical damping of the oscillator as a result of the strong optical spring, which causes the mechanical damping to be negligible in comparison to the damping provided by the optical spring [24].

We may then write, in the frequency domain,

$$
-m \Omega^{2} x=-\frac{2}{c}\left(\frac{\partial P}{\partial a_{1}}\right)_{x} a_{1}-m \Omega_{\mathrm{OS}}^{2} x+F_{\mathrm{ext}},
$$

where $\Omega_{\mathrm{OS}}$ is the optical spring frequency, and we have used the relation $(\partial P / \partial x)_{a_{1}}=m \Omega_{\mathrm{OS}}^{2}$. Solving for $x$ and $\delta P$ :

$x=\frac{1}{m\left(\Omega^{2}-\Omega_{\mathrm{OS}}^{2}\right)} \frac{2}{c}\left(\frac{\partial P}{\partial a_{1}}\right)_{x} a_{1}-\frac{1}{m\left(\Omega^{2}-\Omega_{\mathrm{OS}}^{2}\right)} F_{\mathrm{ext}}$ and

$$
\begin{aligned}
\delta P & =\frac{m c x \Omega^{2}}{2}+\frac{c}{2} F_{\mathrm{ext}} \\
& =\frac{\Omega^{2}}{\Omega^{2}-\Omega_{\mathrm{OS}}^{2}}\left(\frac{\partial P}{\partial a_{1}}\right)_{x} a_{1}-\frac{c}{2} \frac{\Omega_{O S}^{2}}{\Omega^{2}-\Omega_{\mathrm{OS}}^{2}} F_{\mathrm{ext}} .
\end{aligned}
$$

As evident from the first term in Eq. (4), for frequencies far below the optical spring resonance $\left(\Omega \ll \Omega_{\mathrm{OS}}\right)$, the intracavity power fluctuations are reduced by $\left(\Omega_{\mathrm{OS}} / \Omega\right)^{2}$. Measuring the amplitude quadrature of the light in transmission is equivalent to measuring the same quadrature as the intracavity field because the transmitted carrier has the same phase as the intracavity carrier, as depicted in Fig. 1(a). Thus, the amplitude fluctuations in the transmitted field will also be strongly suppressed. The vacuum fluctuations that promptly reflect from the end mirror dominate, and the total noise will be close to shot noise. Therefore, no signature of QBA is observed in this measurement as a result of the strong optical spring. External (classical) forces do couple to the intracavity power and to the amplitude of the transmitted field but are also suppressed by the same factor as the power fluctuations, as seen in Eq. (3). Thus, measuring the amplitude of the transmission does provide a measure of the mirror displacement, but without a component from QBA.

If the cavity is measured in reflection, however, the situation is very different. The intracavity field exiting at the input coupler must mix with the field that promptly reflects from the input coupler. These two fields are at a different phase that depends on the cavity detuning. Measuring the amplitude quadrature of the reflected field will therefore measure a different quadrature than doing so in transmission. Figure 1(a) illustrates the relationship between the intravacity light and the cavity inputs and outputs.

To accurately quantify the backaction cancellation, we use a numerical model using the two-photon formalism [25], with the results shown in Fig. 1(b). Measuring the amplitude in transmission corresponds to measuring at approximately $-90^{\circ}$. This quadrature is sensitive to the position of the microresonator, and the total noise is equal to the quadrature sum of thermal noise and shot noise only, with no contribution from QBA. Using the thermal noise as an example "signal," Fig. 1(c) displays the increase in the signal-to-noise ratio when comparing the transmission and reflection quadratures. It is important to note that the increase in the signal-to-noise ratio is a result of the change in quadrature facilitated by the presence of the optical spring. The increase in SNR is not simply a consequence of the optical spring suppressing the QBA because the optical spring reduces both the signal and noise by the same amount, as mentioned above. In the case of a resonant cavity, without an optical spring, this change in quadrature is not possible without an external homodyne detector.

Figure 1(b) also illustrates that it is possible for the quantum noise to be below the shot noise level. In this case, 
(a)

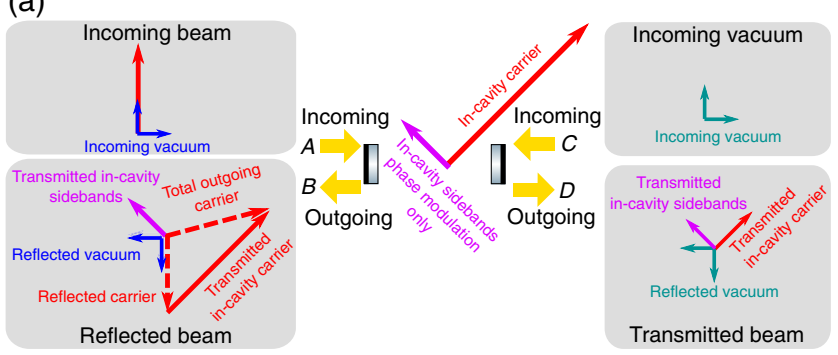

(b)

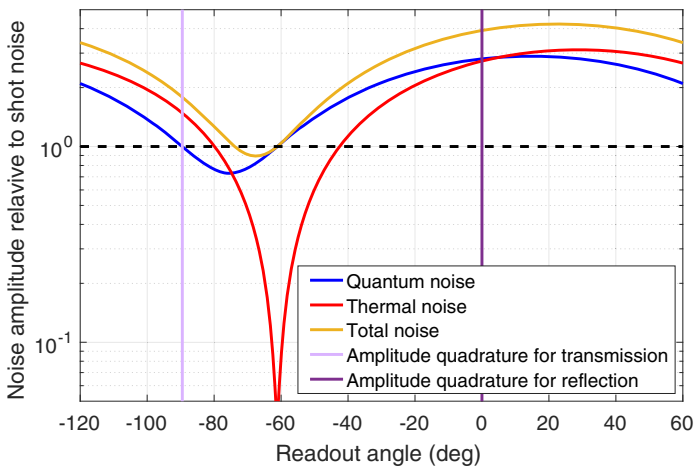

(c)

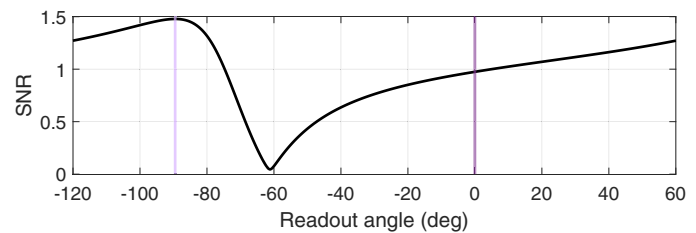

FIG. 1. (a) Diagram showing the relationship between the quadratures of the intracavity light, transmitted light, and reflected light. Input $A$ contains the incoming vacuum and the carrier of the laser field, while input $C$ only consists of vacuum fluctuations. The transmitted beam $D$ is composed of the reflected vacuum fluctuations from $C$ and a small amount of the intracavity field, whose amplitude fluctuations are highly suppressed, as discussed in the text. The reflected light $B$ is made up of the promptly reflected carrier and vacuum fluctuations as well as the outgoing intracavity light. The total reflected carrier is the vector sum of the promptly reflected and transmitted intracavity beams. (b) Modeled quantum noise, thermal noise, and the total noise relative to shot noise (dotted horizontal line) at $20 \mathrm{kHz}$ as a function of readout angle with a cavity circulating power of $155 \mathrm{~mW}$ and detuning of -0.55 linewidths. The amplitude quadrature for light detected in reflection of the cavity is set at $0^{\circ}$ and marked with a vertical line. The amplitude quadrature for the light detected in transmission of the microresonator is marked with the vertical line at $-89^{\circ}$ where the quantum noise curve is equal to shot noise. Note that the figure does not include technical noise sources and is meant to serve more as a pedagogical tool showing that the total noise can be reduced. A detailed noise budget is included later in the paper. (c) The signal-to-noise ratio between the thermal noise and quantum noise demonstrating the increase in SNR in the transmission measurement.

the correlations produced inside the optomechanical cavity create ponderomotive or optomechanically squeezed light. Adding a phase sensitive homodyne readout detector to either the reflection or transmission creates the ability to access this region of optomechanical squeezing [26].
Furthermore, adding the homodyne readout in reflection of the cavity allows the QBA-free measurement presented here to be performed in both reflection and transmission of the cavity. We do not add the homodyne detector in this experiment, however, to avoid adding additional complexity and excess feedback noise and phase noise to the measurement.

\section{EXPERIMENT AND RESULTS}

A comparison of the experimental setups for the measurement in transmission and reflection is shown in Fig. 2. In both setups, intensity stabilized light is injected into the optomechanical cavity, which is formed by a $1 \mathrm{~cm}$ radius of curvature macroscopic mirror and a high-reflectivity single-crystal microresonator. Detailed parameters of the cavity and microresonator are included in Table I and in Refs. [12-17].

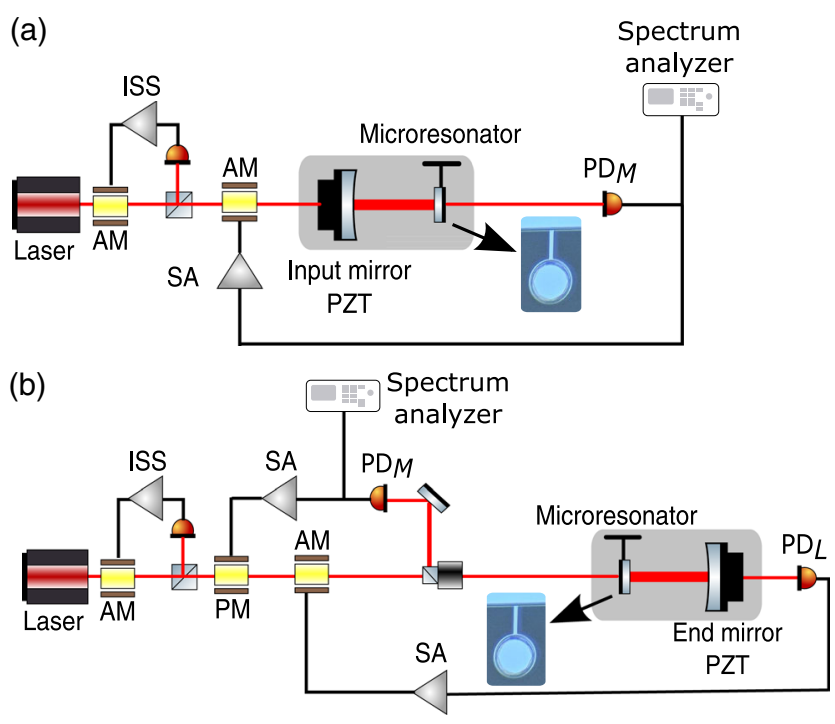

FIG. 2. Experimental setup for the (a) backaction cancellation measurement in transmission of the cavity and (b) benchmark measurement in reflection of the cavity [12]. (a) Light from a $1064 \mathrm{~nm} \mathrm{Nd:YAG} \mathrm{laser} \mathrm{is} \mathrm{passed} \mathrm{through} \mathrm{two} \mathrm{amplitude}$ modulators (AM) before being injected into the optomechanical cavity, which sits on a suspended optical breadboard to reduce seismic motion and is housed in a vacuum chamber at $10^{-7}$ Torr (shown in shaded gray). A micrograph of the single-crystal microresonator, comprising a 70- $\mu$ m-diameter GaAs/AlGaAs mirror pad supported by a GaAs cantilever, is included in the diagram. An intensity stabilization servo (ISS) is used to stabilize the laser power to shot noise by feeding back to the first AM. The light transmitted through the optomechanical cavity is detected by photodetector $\mathrm{PD}_{M}$. The signal from $\mathrm{PD}_{M}$ is sent through a servo amplifier (SA) before being sent to the second $\mathrm{AM}$ to lock the cavity. The signal from $\mathrm{PD}_{M}$ is also sent to a spectrum analyzer for further analysis. PZT refers to a piezoelectric transducer or specifically lead zirconate titanate $\left[\mathrm{Pb}\left(\mathrm{Zr}_{x} \mathrm{Ti}_{1-x}\right) \mathrm{O}_{3}\right]$ which is attached to the macroscopic mirror. 
TABLE I. Properties of the microresonator and experimental parameters determined from measurements. The second half of the table has the common optomechanical parameters for comparison with the current state-of-the-art optomechanical systems. As explained in the Methods section of Ref. [12], these parameters are used to characterize an on-resonance system and are not used in our calculations.

\begin{tabular}{ll}
\hline \hline Microresonator mirror pad diameter & $70 \mu \mathrm{m}$ \\
Cantilever length & $55 \mu \mathrm{m}$ \\
Cantilever width & $8 \mu \mathrm{m}$ \\
Cantilever thickness & $220 \mathrm{~nm}$ \\
Microresonator mass & $50 \mathrm{ng}$ \\
Microresonator fundamental frequency & $876 \mathrm{~Hz}$ \\
Microresonator quality factor & 16000 \\
Microresonator transmission & $250 \mathrm{ppm}$ \\
Macroscopic mirror transmission & $50 \mathrm{ppm}$ \\
Cavity losses (transmission) & $200 \pm 10 \mathrm{ppm}$ \\
Cavity losses (reflection) & $180 \pm 10 \mathrm{ppm}$ \\
Cavity linewidth HWHM $(\gamma)$ & $580 \mathrm{kHz}$ \\
Cavity detuning (transmission) & $0.50 \pm 0.05 \gamma$ \\
Cavity detuning (reflection) & $0.55 \pm 0.05 \gamma$ \\
Intracavity power & $155 \pm 10 \mathrm{~mW}$ \\
Optical spring frequency $\left(\Omega_{\mathrm{OS}}\right)$ & $142 \mathrm{kHz}$ \\
Optomechanical single-photon & $2 \pi \times 380 \mathrm{kHz}$ \\
coupling strength & \\
Linearized light-enhanced & $2 \pi \times 3.5 \mathrm{MHz}$ \\
optomechanical coupling & $8 \times 10^{7}$ \\
Photon number circulating in cavity & $7.4 \times 10^{8}$ \\
Multiphoton cooperativity & $7.4 \times 10^{9}$ \\
Thermal phonon occupation & \\
\hline \hline
\end{tabular}

We perform two displacement measurements of the microresonator, one in transmission of the cavity, as shown in Fig. 2(a), and one in reflection of the cavity, as shown in Fig. 2(b). We first perform a displacement noise measurement with the light injected on the microresonator side of the cavity and detected in reflection. This provides us with a measurement of the displacement noise when the contribution from QBA is included. The cavity orientation and feedback using the phase modulator (PM) for this measurement is shown in Fig. 2(b) and mimics that of the QBA measurement in Ref. [12]. To determine the optical parameters including the detuning, circulating power, and intracavity loss for the measurement, we have found that the most accurate method is using measurements of the optical spring [12]. We measure the optical spring frequency to be $142 \mathrm{kHz}$ in this orientation. The reflection measurement is performed with an input power of $50 \mu \mathrm{W}$ and a detuning of $0.55 \pm 0.05$ linewidths. The measurement of the optical spring, along with the cavity detuning and input power, was then used to calculate the circulating power of $155 \pm 10 \mathrm{~mW}$ inside the cavity and the intracavity loss to be $200 \pm 10 \mathrm{ppm}$.

After performing the reflection measurement, we switch which side of the cavity the light is incident on and inject the light from the macroscopic mirror side of the cavity.
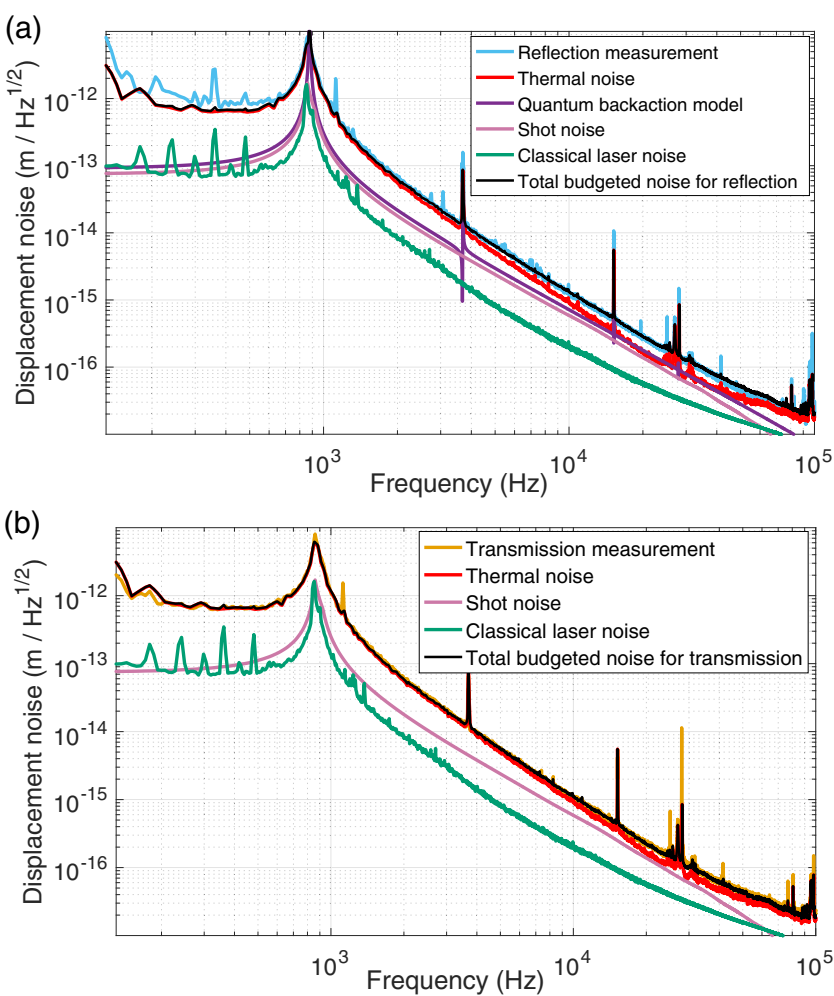

FIG. 3. Measured and budgeted displacement noise spectra taken in reflection (a) and transmission (b) of the cavity. The individual noise sources for each measurement are added in quadrature to calculate the total budgeted noise. The coupling of the higher order mechanical modes of the microresonator are reduced with respect to their magnitudes in Ref. [12] as a result in improvements in our ability to align the optical beam onto the nodal points of those modes.

Detecting the amplitude quadrature of the light in transmission with photodetector $\mathrm{PD}_{M}$ allows us to have a QBA-free measurement of the microresonator's position. Between the two measurements, we carefully keep the circulating power and detuning as close to constant as experimentally possible so that we can make a fair comparison and look for a reduction in the measured displacement noise. We perform the transmission measurement with the same optical spring frequency as the reflection measurement and keep the other cavity parameters as close as experimentally possible between the two measurements, as seen in Table I. The intracavity loss is slightly different between the two measurements as a result of the strong dependence of cavity loss on the nanometerlevel positioning of the optical mode on the microresonator.

The total noise measured in reflection and transmission of the cavity is shown in Fig. 3 along with the individual noise sources measured in the lab and the expected contribution from QBA based on input-output relations using the measured values in Table I $[12,25]$. The residual backaction in the transmission measurement adds $1 \%$ or less to the total measured noise across the entire frequency 

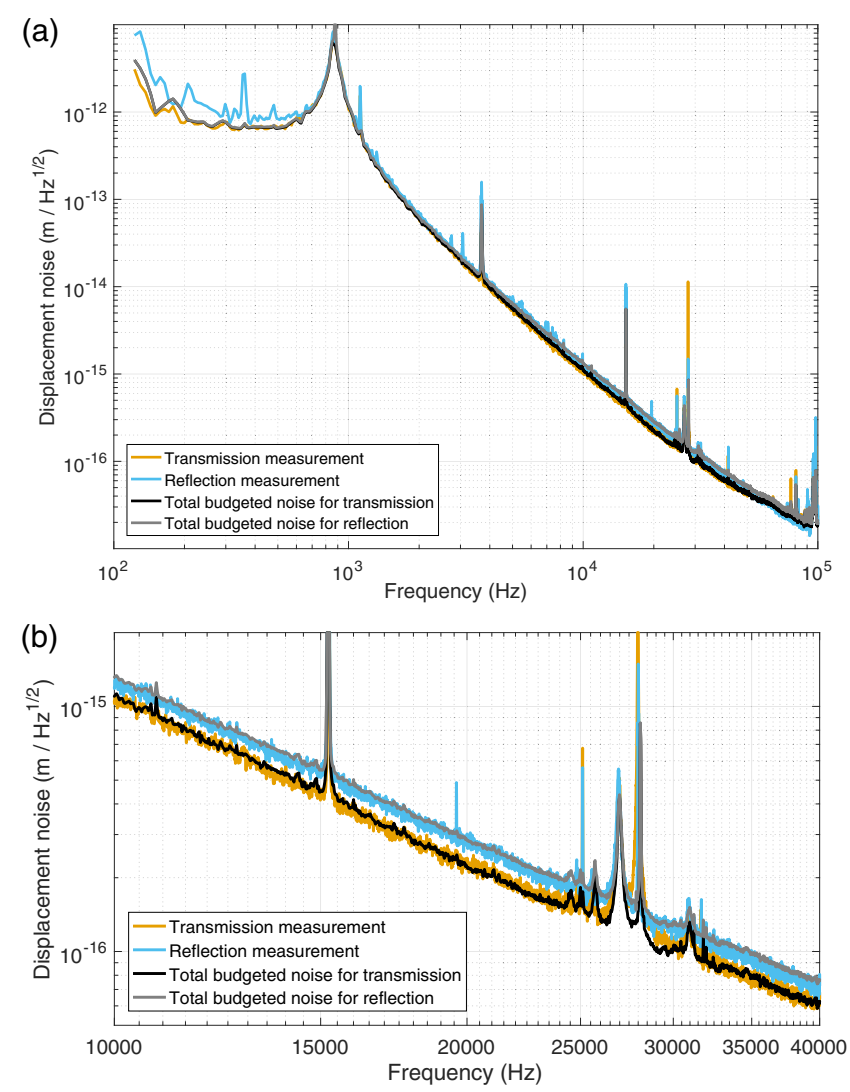

FIG. 4. Measured and budgeted total noise for the transmission and reflection measurements across the entire measurement bandwidth (a) and the frequency range where the backaction cancellation is largest, with a maximum reduction in the total displacement noise of $2 \mathrm{~dB}$ (b). The total budget noise for each measurement consisting of the sum of the individual noise sources shown in Fig. 3.

band and is thus not shown in Fig. 3(b). The measurements in Fig. 3 and throughout the paper are shown with the microresonator treated as a free mass with its natural resonance frequency unaltered by the strong optical spring. We remove the effects of the optical spring because it is treated as a feedback mechanism for controlling the cavity [24].

To see the difference between the two noise measurements more clearly, we include a magnified version of the noise budgets in Fig. 4, where we focus on the region between 10 and $40 \mathrm{kHz}$ and plot the total measured and budgeted noise for each measurement. As shown in Figs. 3 and 4 , the measured noise for the transmission measurement is reduced between 2 and $50 \mathrm{kHz}$, with the maximum reduction of $20 \%$ or about $2 \mathrm{~dB}$ at $20 \mathrm{kHz}$ as compared to the reflection measurement. This reduction in noise is consistent with Eq. (4), with the backaction being reduced by a factor of $\left(\Omega_{\mathrm{OS}} / \Omega\right)^{2} \approx 50$ at a measurement frequency of $20 \mathrm{kHz}$. The frequency range over which we observe the reduction in displacement noise reduction is limited to the region in which QBA contributes a measurable amount of noise to the total noise budget. As we are limited by thermal noise at room temperature, lowering the thermal noise level by cryogenically cooling the microresonator would increase both the amount that the noise is reduced and the frequency range over which we observe the backaction cancellation.

\section{QUANTUM-NOISE-FREE THERMAL NOISE MEASUREMENT}

We have previously shown that the measurement carried out in reflection is dominated by QBA by performing measurements at multiple levels of intracavity power and observing how the displacement noise scales as a function of power [12]. To further confirm that we are canceling the QBA in the method presented here, we modify the transmission measurement by splitting the transmitted light across two photodetectors, and cross-correlating their outputs to remove the effects of shot noise. The results of this measurement are shown in Fig. 5, and details of the crosscorrelation measurement are included as follows.

First, to eliminate QBA, we measure the intensity of the transmitted light, which is insensitive to QBA as explained above. Then, we split the light transmitted through the cavity onto two photodetectors using a beam splitter. By taking advantage of the fact that thermal noise is correlated between the two detectors, whereas shot noise is not, we can measure only the thermal noise by looking at the crosspower spectral density between the two photocurrents. The optical field in the amplitude quadrature for the first and second PD may be written as

$$
\begin{aligned}
& a_{1}=v_{1}+\alpha x_{\mathrm{th}}, \\
& b_{1}=v_{2}+\alpha x_{\mathrm{th}},
\end{aligned}
$$

where $v_{1}$ and $v_{2}$ correspond to uncorrelated vacuum fluctuations and $\alpha$ is the coupling constant for thermal motion. A cross-power spectral density taken between the two outputs of the PDs gives

$$
\left|\left\langle S_{12}\right\rangle\right|=|\alpha|^{2} S_{\mathrm{th}}
$$

where $S_{\mathrm{th}}$ is the thermal noise spectrum. Notably, all the terms involving vacuum fluctuations go to 0 because they are uncorrelated with each other and with the thermal noise. In principle, this allows for the true thermal noise spectrum to be obtained, with no contribution from quantum noise.

In our experiment, however, we require the transmitted signal to be fed back to an AM for locking the cavity, which can create additional correlations. In this case, the feedback to the cavity reinjects the noise on only one of the PDs back onto the amplitude quadrature of the input light. In this case, the fields for each PD may be written as 


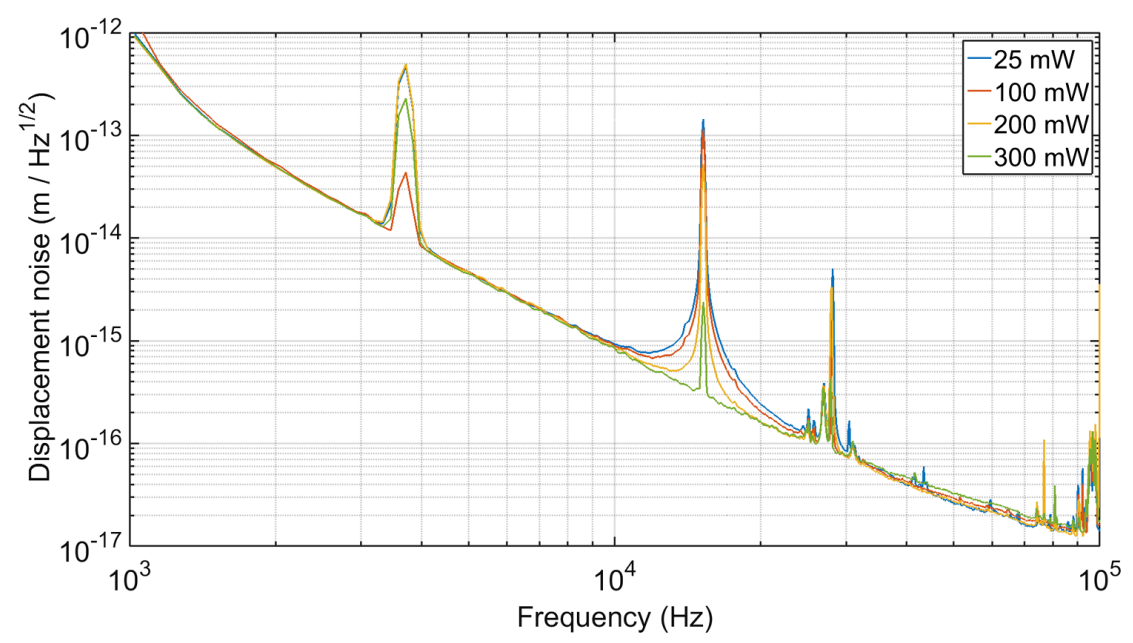

FIG. 5. Quantum-noise-free thermal noise spectrum measured at four different power levels. The measurement is performed by splitting the transmitted light onto two photodetectors and cross-correlating their outputs. The measured thermal noise remains constant across the range of powers at frequencies away from the resonances of the higher order modes. At frequencies near the higher order modes, the measured noise changes as a result of the change in cavity alignment due to the deflection of the cantilever by radiation pressure [12]. Realigning the beam at high power recovers the same thermal noise as measured with low power. There is no signature of QBA as expected.

$$
\begin{aligned}
a_{1} & =v_{1}+\alpha x_{\mathrm{th}}-\frac{G}{1+G}\left(v_{1}+\alpha x_{\mathrm{th}}\right) \\
& =\frac{v_{1}+\alpha x_{\mathrm{th}}}{1+G}, \\
b_{1} & =v_{2}+\alpha x_{\mathrm{th}}-\frac{G}{1+G}\left(v_{1}+\alpha x_{\mathrm{th}}\right) \\
& =v_{2}+\frac{\alpha x_{\mathrm{th}}}{1+G}-\frac{G}{1+G} v_{1},
\end{aligned}
$$

where $G$ is the open loop gain for the feedback loop [24]. Then the cross-power spectral density between the two photocurrents is

$$
\left|\left\langle S_{12}\right\rangle\right|=\frac{|\alpha|^{2} S_{\mathrm{th}}}{1+G}+\frac{G}{(1+G)^{2}},
$$

where we have normalized the power spectral density to shot noise. Therefore, there is an additional noise contribution equal to shot noise multiplied by the factor $G /(1+G)$. For this extra noise to be significant, shot noise must be similar to or greater in magnitude than thermal noise, which only occurs at the high power levels. In addition, the loop gain would need to satisfy $|G| \gtrsim 1$. For the frequencies of interest here, these requirements are not simultaneously satisfied, and the extra noise is small.

Performing the cross-correlation and removing the effects of shot noise allows us to compare measurements taken with different circulating power levels and demonstrate that the measured noise remains constant and at the level of thermal noise. If QBA were present in the measurements, we would expect to see an increase in the measured noise in Fig. 5 that scales with the square root of power [5].

In addition to confirming that the QBA is canceled in the measurements, measuring the cross correlation also allows us to perform a quantum noise-free measurement of the thermal noise of the microresonator by averaging the uncorrelated shot noise to zero. This measurement also serves as a validation that the temperature of the microresonator does not significantly increase between low and high circulating power measurements. Physical heating of the device would lead to an increase in thermal noise, which scales with the square root of temperature. The results shown in Fig. 5 reveal that there is not an increase in thermal noise for measurements spanning a factor of 10 in circulating power.

\section{EFFECT OF UNCERTAINTIES}

We include uncertainties for the experimental parameters listed in Table I. While the individual uncertainties are on the order of 5\%-10\%, they do not limit our ability to measure the $20 \%$ reduction in measured displacement noise between the transmission and reflection measurements because we directly compare two calibrated displacement measurements. As discussed in detail in the Methods section of Ref. [12], we calibrate the measurement performed at the photodetector into units of displacement by modulating the laser frequency and measuring its frequency-dependent response at the measurement PD. This measurement relates a change in laser frequency to a change in cavity length and allows us to measure the effect of a given amount of displacement on the measurement PD. By calibrating the measurements in units of 
$\mathrm{m} / \sqrt{\mathrm{Hz}}$, the comparison between measurements is no longer limited to uncertainties in mass, power, detuning, and loss because those values are not used in the calibration process. In addition, a systematic uncertainty in the calibration of the laser piezoelectric tuning element does not limit our ability to measure a reduction in noise as a result of it being common to both measurements. More details of the calibration method are included in the Methods section of Ref. [12].

\section{CONCLUSION}

We have performed two displacement noise measurements of a microresonator in an optomechanical cavity and have shown that the measured noise is reduced when the light is detected in transmission of the cavity as compared to in reflection of the cavity. The reduction in noise is a result of the cancellation of QBA in the spectrum of the transmitted light, which arises as a consequence of the strong optical spring. The measured noise is reduced between 2 and $50 \mathrm{kHz}$ by up to $2 \mathrm{~dB}$ and is consistent with the theoretical calculations and the measurements of the individual noise sources. To confirm the QBA cancellation, we perform a cross-correlation with two photodetectors in transmission of the cavity and show that the measured noise remains constant for measurements spanning a factor of 12 in power. Our results demonstrate the cancellation of QBA in the audio band at frequencies relevant to current and future interferometric gravitational wave detectors. The successful removal of QBA from the measurement also paves the way for future measurements at or below the standard quantum limit. We are currently a factor of approximately 4 above the standard quantum limit at room temperature and will be able to reduce the currently limiting thermal noise by cryogenically cooling the microresonator.

\section{ACKNOWLEDGMENTS}

The microresonator manufacturing was carried out at the UCSB Nanofabrication Facility. This document has been assigned LIGO Document No. LIGO-P1800380. T. Corbitt, J. C., and T. Cullen acknowledge support by National Science Foundation Grants No. PHY-1150531 and No. PHY-1806634. Y. C. was funded by NSF Grants No. PHY-1708212 and No. PHY-1612816 and Simons Foundation Grant No. 568762.

Y.C. conceptualized the theoretical background of the experiment. Y. C., T. Corbitt, and J. C. planned the experiment. J. C. led the investigation with help from T. Cullen and T. Corbitt, who also provided oversight. P. H., D. F., and G.D.C. fabricated the microresonators. J.C. led the writing of the original draft, and all authors assisted in the review and editing of the final draft.

The authors declare no competing interests.
[1] A. Michelson and E. Morley, On the Relative Motion of the Earth and the Luminiferous Ether, Am. J. Sci. 34, 333 (1887).

[2] B. P. Abbott et al. (LIGO Scientific Collaboration and Virgo Collaboration), Observation of Gravitational Waves from a Binary Black Hole Merger, Phys. Rev. Lett. 116, 061102 (2016).

[3] B. P. Abbott et al. (LIGO Scientific Collaboration and Virgo Collaboration), GW170817: Observation of Gravitational Waves from a Binary Neutron Star Inspiral, Phys. Rev. Lett. 119, 161101 (2017).

[4] B. P. Abbott et al. (LIGO Scientific Collaboration and Virgo Collaboration), GWTC-1: A Gravitational-Wave Transient Catalog of Compact Binary Mergers Observed by LIGO and Virgo During the First and Second Observing Runs, Phys. Rev. X 9, 031040 (2019).

[5] C. M. Caves, Quantum-Mechanical Radiation-Pressure Fluctuations in an Interferometer, Phys. Rev. Lett. 45, 75 (1980).

[6] V. B. Braginsky and A. B. Manukin, Measurement of Weak Forces in Physics Experiments (University of Chicago Press, Chicago, 1977).

[7] C. M. Caves, Quantum-Mechanical Noise in an Interferometer, Phys. Rev. D 23, 1693 (1981).

[8] T. P. Purdy, R. W. Peterson, and C. A. Regal, Observation of Radiation Pressure Shot Noise on a Macroscopic Object, Science 339, 801 (2013).

[9] J. D. Teufel, F. Lecocq, and R. W. Simmonds, Overwhelming Thermomechanical Motion with Microwave Radiation Pressure Shot Noise, Phys. Rev. Lett. 116, 013602 (2016).

[10] T. P. Purdy, K. E. Grutter, K. Srinivasan, and J. M. Taylor, Quantum Correlations from a Room-Temperature Optomechanical Cavity, Science 356, 1265 (2017).

[11] V. Sudhir, R. Schilling, S. A. Fedorov, H. Schütz, D. J. Wilson, and T. J. Kippenberg, Quantum Correlations of Light from a Room-Temperature Mechanical Oscillator, Phys. Rev. X 7, 031055 (2017).

[12] J. Cripe, N. Aggarwal, R. Lanza, A. Libson, R. Singh, P. Heu, D. Follman, G. D. Cole, N. Mavalvala, and T. Corbitt, Measurement of Quantum Back Action in the Audio Band at Room Temperature, Nature (London) 568, 364 (2019).

[13] G. D. Cole, S. Gröblacher, K. Gugler, S. Gigan, and M. Aspelmeyer, Monocrystalline $\mathrm{Al}_{x} \mathrm{Ga}_{1-x}$ As Heterostructures for High-Reflectivity High-Q Micromechanical Resonators in the Megahertz Regime, Appl. Phys. Lett. 92, 261108 (2008).

[14] G. D. Cole, Cavity Optomechanics with Low-Noise Crystalline Mirrors, in Optical Trapping and Optical Micromanipulation IX, SPIE Proceedings Vol. 8458 (SPIE-International Society for Optical Engineering, Bellingham, WA, 2012), p. 845807.

[15] G. D. Cole, W. Zhang, M. J. Martin, J. Ye, and M. Aspelmeyer, Tenfold Reduction of Brownian Noise in High-Reflectivity Optical Coatings, Nat. Photonics 7, 644 (2013).

[16] G. D. Cole, W. Zhang, B. J. Bjork, D. Follman, P. Heu, C. Deutsch, L. Sonderhouse, J. Robinson, C. Franz, A. Alexandrovski, M. Notcutt, O. H. Heckl, J. Ye, and M. Aspelmeyer, High-Performance Near- and Mid-Infrared Crystalline Coatings, Optica 3, 647 (2016). 
[17] R. Singh, G. D. Cole, J. Cripe, and T. Corbitt, Stable Optical Trap from a Single Optical Field Utilizing Birefringence, Phys. Rev. Lett. 117, 213604 (2016).

[18] C. F. Ockeloen-Korppi, E. Damskägg, J.-M. Pirkkalainen, A. A. Clerk, M. J. Woolley, and M. A. Sillanpää, Quantum Backaction Evading Measurement of Collective Mechanical Modes, Phys. Rev. Lett. 117, 140401 (2016).

[19] C. B. Møller, R. A. Thomas, G. Vasilakis, E. Zeuthen, Y. Tsaturyan, M. Balabas, K. Jensen, A. Schliesser, K. Hammerer, and E.S. Polzik, Quantum Back-ActionEvading Measurement of Motion in a Negative Mass Reference Frame, Nature (London) 547, 191 (2017).

[20] H. J. Kimble, Y. Levin, A. B. Matsko, K. S. Thorne, and S. P. Vyatchanin, Conversion of Conventional Gravitational-Wave Interferometers into Quantum Nondemolition Interferometers by Modifying Their Input and/or Output Optics, Phys. Rev. D 65, 022002 (2001).

[21] S. Vyatchanin and E. Zubova, Quantum Variation Measurement of a Force, Phys. Lett. A 201, 269 (1995).
[22] N. S. Kampel, R. W. Peterson, R. Fischer, P.-L. Yu, K. Cicak, R. W. Simmonds, K. W. Lehnert, and C. A. Regal, Improving Broadband Displacement Detection with Quantum Correlations, Phys. Rev. X 7, 021008 (2017).

[23] T. Corbitt, Y. Chen, E. Innerhofer, H. Müller-Ebhardt, D. Ottaway, H. Rehbein, D. Sigg, S. Whitcomb, C. Wipf, and N. Mavalvala, An All-Optical Trap for a Gram-Scale Mirror, Phys. Rev. Lett. 98, 150802 (2007).

[24] J. Cripe, N. Aggarwal, R. Singh, R. Lanza, A. Libson, M. J. Yap, G. D. Cole, D. E. McClelland, N. Mavalvala, and T. Corbitt, Radiation-Pressure-Mediated Control of an Optomechanical Cavity, Phys. Rev. A 97, 013827 (2018).

[25] T. Corbitt, Y. Chen, and N. Mavalvala, Mathematical Framework for Simulation of Quantum Fields in Complex Interferometers Using the Two-Photon Formalism, Phys. Rev. A 72, 013818 (2005).

[26] N. Aggarwal, T. J. Cullen, J. Cripe, G. D. Cole, R. Lanza, A. Libson, D. Follman, P. Heu, T. Corbitt, and N. Mavalvala, Room-Temperature Optomechanical Squeezing, Nat. Phys. 16, 784 (2020). 